\title{
Role of N6-methyladenosine Regulated IncRNAs in Prognosis, Tumor Microenvironment and Immune Checkpoints of Pancreatic Ductal Adenocarcinoma
}

\section{Guangzhen Qu}

Capital Medical University

\section{Dong Wang}

Capital Medical University

Weiyu Xu

Capital Medical University

Wei Guo ( $\nabla$ guowei@ccmu.edu.cn )

Capital Medical University

\section{Research Article}

Keywords: Pancreatic ductal adenocarcinoma, tumor microenvironment, immunotherapy, N6methyladenosine, IncRNA, immune infiltration, PD-L1

Posted Date: December 3rd, 2021

DOI: https://doi.org/10.21203/rs.3.rs-1107862/v1

License: (c) (i) This work is licensed under a Creative Commons Attribution 4.0 International License. Read Full License 


\section{Abstract}

\section{Background}

The purpose of this study was to explore the correlation between N6-methyladenosine (m6A)-regulated IncRNAs and tumor prognosis, immune infiltration, immune checkpoints (ICPs) expression in pancreatic ductal adenocarcinoma (PDAC).

\section{Methods}

We downloaded the raw RNA-sequence data and clinical data of PDAC from https://xenabrowser.net/ (cohort: TCGA Pancreatic Cancer) and Genotype-Tissue Expression project (GTEx). The m6A-regulated IncRNA was obtained by co-expression analysis. After that, IncRNA profiles and PDAC survival information were merged, and m6A-regulated multi-IncRNA prognostic model was constructed through least absolute shrinkage and selection operator (LASSO) analysis. Through consensus clustering algorithm analysis, PDAC samples were divided into $\mathrm{C} 1$ and $\mathrm{C} 2$ groups. The downstream pathway signals of the two groups were constructed by Gene set enrichment analysis (GSEA) analysis. Finally, we detect the links between m6A regulated IncRNAs, immune infiltration, immune checkpoint gene expression.

\section{Results}

A total of 28 differential expressed m6A-regulated IncRNAs were identified, and based on this, a total of two subtypes of PDAC were obtained. A risk score nomogram consist of 11 m6A-regulated IncRNAs was constructed based on LASSO regression analysis. PDAC patients were divided into low-risk and high-risk groups based on risk scores. In addition to that, we identified ID01 as a potential novel ICPs in PDAC.

\section{Conclusion}

This study demonstrates an indispensable role for m6A-regulated IncRNAs in the tumor microenvironment and immune infiltration. We could screen patients suitable for immunotherapy. Long term survival of PDAC patients can be predicted by 11 m6A regulated IncRNAs superiorly. The immune infiltration and ICPs expression were further explored in both groups.

\section{Introduction}

Pancreatic ductal adenocarcinoma (PDAC) is a notorious tumor of extremely high malignancy, which is the seventh leading cause of cancer death by 2020 , with the highest incidence in Europe, North America, and Australia / New Zealand ${ }^{[1]}$. It has been pointed out that PDAC is projected to surpass breast cancer as the third most tumor lethal disease by $2025^{[2]}$. The only curable treatment of PDAC is surgical resection, but this results in a 5-year overall survival rate of only $9 \%$ due to the fact that most patients have lost surgical opportunity when diagnosed with PDAC ${ }^{[3]}$. The advancement of adjuvant chemotherapy has modestly improved the long-term survival of these patients. However, mutations like 
KRAS and unique heterogeneous tumor microenvironment (TME) characterized by a fibro-inflammatory stroma that contributes to disease progression, a part of PDAC patients suffered from chemotherapy resistance ${ }^{[4,5]}$. In recent years, immunotherapy has received extensive attention and become a promising star in tumor therapy. Immune checkpoint blockade (ICBs) targeting ICPs molecules such as ipimab, pabolizumab and atzumab have shown encouraging effects in many solid tumors. However, the efficacy of PD-1/ PD-L1 based immunotherapy for PDAC still needs further exploration, and it is urgent to exploit new immune checkpoints for PDAC.

RNA modification confers upon tumor cells the ability to rapidly and inversely alter after transcription to allow them to adapt and survive in the rapidly changing tumor microenvironment (TME) ${ }^{[6]}$. Of more than 170 RNA modifications, N6-methyladenosine (m6A) modifications are the most common and most extensively studied post-transcriptional modifications of RNA ${ }^{[6]}$. Long non coding RNAs (IncRNAs) are non-coding RNAs greater than 200 nucleotides in length and are vital for pathogenesis of cancers ${ }^{[7]}$. LncRNAs play essential roles at the transcriptional / post transcriptional level and chromatin modification by regulating gene expression ${ }^{[8]}$.M6A can regulate IncRNA structure through binding sites for $\mathrm{m} 6 \mathrm{~A}$ readers, this may allow specific RNA binding proteins to access m6A residues. Similarly, m6A can also affect the function of IncRNAs through the ceRNA network. For example, FAM225a, a IncRNA modified by m6A regulation, acts as a sponge for mir-590-3p and mir-1275 in nasopharyngeal carcinoma ${ }^{[9]}$. A study concluded that m6A reader IGF2BP2 can promote stemness-like properties of PDAC through regulates IncRNA DANCR ${ }^{[10]}$. However, the full function of m6A and its regulated IncRNAs in PDAC still needs further elaboration.

The PDAC tumor microenvironment contains a large proportion of dense desmoplasia and immunosuppressive cell populations, which limit cytotoxic $T$ responses ${ }^{[11]}$. Cancer associated fibroblasts (CAFs) contribute to the fibroblast proliferative and generate immunosuppressive TME by producing extracellular matrix proteins and cytokines, as well as promote tumor cell growth ${ }^{[12]}$. PD-L1 on the surface of PDAC parenchymal tumor cells and PD-1 of T cells can be important mechanism for immunotherapy. However, ICBs targeting PD-L1 have limited effects in PDAC due to an attenuated tumor immunogenic and immunosuppressive TME ${ }^{[13]}$.

Yuan and $\mathrm{Hu}{ }^{[14,15]}$ previously constructed a prognostic signaling pathway based on m6A regulated IncRNAs in pancreatic ductal adenocarcinoma, but in Yuan's study, the shortcoming is lack of detailed exploration of immune microenvironment in PDAC and further exploration of immune infiltration. Hu's study started from m6A regulated IncRNAs shared by TCGA and ICGC, PDAC samples were not clustered and potential therapeutic targets were not further explored. In addition, some samples were additionally removed, which has the potential to increase bias. In our study, we promulgate the association of m6Aregulated IncRNAs with tumor prognosis, TME, immune infiltration, and expression of related ICPs genes in PDAC patients. we defined a $11 \mathrm{~m} 6 \mathrm{~A}$ regulated IncRNAs that effectively predicted the prognosis of patients with PDAC. 


\section{Results}

\section{Expression of m6A RNA methylation regulators and m6A-regulated IncRNAs in PDAC}

The m6A RNA methylation regulators play an indispensable role in tumor occurrence, proliferation, invasion and metastasis. We probe and systematically download the $23 \mathrm{~m} 6 \mathrm{~A}$ regulator genes expression and IncRNA expression of 178 tumor samples and 4 adjacent paracancerous samples from TCGA database and 167 normal samples from GTEx database. All the expressions of 23 m6A RNA methylation regulator genes differed between tumor and normal samples $(P<0.01)$.

From the heatmap and boxplot (Figure S1A and Figure S1B) of 23 m6A RNA methylation regulator genes expression, it can be observed that "writers" (ZC3H13, RBM15, RBM15B), "readers" (YTHDC1, YTHDC2, YTHDF1, YTHDF2, YTHDF3, IGFBP1, IGFBP3), and "erasers" (ALKBH5) were up-regulated in tumor tissues compared to normal tissues. The remaining $\mathrm{m} 6 \mathrm{~A}$ regulator genes are highly expressed in normal tissues compared with tumor tissues. To further identify the relationship between m6A methylation regulators and IncRNAs, Pearson correlation coefficient and RNA-sequence data was used to select the m6Aregulated IncRNAs. Finally, a total of 1411 IncRNAs with absolute correlation coefficient $>0.4$ and $\mathrm{P}<0.05$ was identified as IncRNAs coexpressed with m6A. (Table S1). Through univariate Cox analyses (Figure 2A), 28 m6A-regulated IncRNAswere obtained.. Among that, 18 m6A-regulated IncRNAs were protective genes, $10 \mathrm{~m} 6 \mathrm{~A}$-regulated IncRNAs were risk genes. . After that, we compared the expression differences of 28 m6A-regulated IncRNAs between tumor and control groups in TCGA database combined with GTEx database, and the visualized heatmaps and boxplots are shown in Figure 2B. The LINC01559, LINC00941, LINC01705, CASC8, AP000695.2 and SH3PXD2A-AS1 tend to beupregulated sharply in tumor tissues.

\section{Correlation between consensus clustering analysis of m6A-regulated IncRNAs and survival of PDAC patients}

Concordance clustering analysis was performed on PDAC samples, which were identified in the cumulative distribution function (CDF), $\mathrm{k}$ takes values ranging from 2-9 and $\mathrm{k}$ denotes cluster counting (Figure S2). According to similarity of m6A-regulated IncRNAs expression and ambiguous proportion for clustering measurements, the optimal clustering parameter was $k=2$ (Figure $3 A$ ) We merged the survival information and target m6A-regulated IncRNAs expression data of PDAC patients. A total of 177 PDAC patients were grouped into Cluster $1(n=87)$ and Cluster $2(n=90)$ based on the m6A-regulated IncRNAs expression. Comparing the clinical variables and demographic variables between Cluster 1 and Cluster 2 according to 28 m6A-regulated IncRNAs, it was found that there was no difference, but there was a significant difference in the overall survival between the two groups. The overall survival of Cluster 1 was superior to that of Cluster $2(P<0.001)$ (Figure 3B).

Correlation between PD-L1 and other immune checkpoints gene expression and m6A-related IncRNAs in Cluster 1 and Cluster 2 
To explore the relationship between m6A-regulated IncRNAs and immune checkpoints (ICPs) gene expression, we compared the distribution of ICPs gene between tumor group and paracancerous cohort and between Cluster 1 and Cluster 2(Figure 7A-H). There was no difference in PD-L1 gene expression between Cluster 1 and Cluster 2 as well as tumor tissues and normal tissues. (Figure 7A, B). However, the expression of the CD47 $(P<0.05)$ (Figure 7C), CD276 ( $P<0.001)$ (Figure 7E), IDO-1 $(P<0.001)$ (Figure 7G) genes that potential be ICPs was statistically different between the tumor and normal groups, showing significantly upregulated expression in the tumor tissue compared to the normal tissue. Meanwhile, the CD276 (B7-H3) gene also showed expression discrepancy in Cluster 1 and Cluster $2(P<0.001)$ (Figure 7F). Co-expression analysis of the genes showed a positive correlation between PD-L1 expression and m6A-regulated IncRNA U62317.1 and LINC01705 (Figure 7I).

\section{Stratification analysis of the m6A-related IncRNAs in immune infiltration and tumor microenvironment}

The tumor microenvironment (TME) is complex and constantly evolving. In addition to stromal cells, fibroblasts, and endothelial cells, the TME contains immune cells ${ }^{[24]}$. Stromal cells in the TME may secrete growth factors, which may not only promote tumor cell growth, but may also chemoattract other cells to migrate into the TME. Immune cells like natural killer and T cells, which can eliminate tumor cells, portend a promising outcome. Researchers have endeavored to understand the characteristics of the TME and the status of immune cells and their interactions with tumor cells. Certain antibody therapies have demonstrated having the ability to activate a patient's own immune system to kill tumor cells ${ }^{[25]}$. We further evaluate the association between m6A-regulated IncRNAs and tumor immune infiltration. Stromal and infiltration level of 22 immune cells was evaluated by calculating the stromal, immune and ESTIMATE score using CIBERSORT. In the subtyping of PDAC samples based on 28-m6A regulated IncRNAs, it can be seen that B cells naive, B cells memory, Plasma cells, CD8 T cells, T cells CD4 memory resting, Monocytes, Macrophages M0, and Dendritic cells activated in Cluster 1 and Cluster 2 have differential distributions (Figure 8A). In Cluster 1, B cells naive, plasma cells, CD8 T cells and Monocytes were infiltrated at higher levels in the TME, whereas in Cluster 2, expression levels of B cells memory, $T$ cells CD4 memory resting and Macrophages M0 were upregulated (Figure 8A). The Cluster 1 had higher stromal score, immune score and estimate score, implicated lower tumor purity (Figure 8B, C, D). All of these scores have statistically significant $(P<0.001)$.

\section{Gene Set Enrichment Analyses (GSEA)}

The Gene Set Enrichment Analyses (GSEA) was performed to explore the signature of m6A regulated IncRNAs in Cluster 1 and Cluster 2. Filtering criteria were FDR $<0.05$ to find notable enriched pathways. As shown in Figure 9, the obvious enriched signature pathway in Cluster 2 was "cell cycle" in Gene Set Enrichment Anaylses (GSEA). Unfortunately, no significant signature enrichment was found in Cluster 1.

\section{Construction of m6A-regulated IncRNAs prognostic nomogram}

The 177 PDAC samples with survival time and status in TCGA were divided into a train set $(\mathrm{N}=108)$ and a test set $(\mathrm{N}=69)$ according to $6: 4$ proportion. Based on the $28 \mathrm{~m} 6 \mathrm{~A}$-regulated IncRNAs as well as the 
LASSO regression analysis (Figure S3), we finally obtained 11 m6A-regulatedlated IncRNAs correlated with prognosis in train set. The risk score for each patient in train and sets was calculated as follows: risk score $=$ U62317. $1 * 0.340993840+$ LINC00941 * $0.120489300+$ AL139287. $1 *(-0.173219714)+$ AC012615.1* $(-0.075084474)+C A S C 8 * 0.229254017+Z 97832.2 *(-0.279993778)+A C 099778.1 *$ $(-0.037908532)+A C 245041.2 * 0.058969635+$ AC091057. $1 * 0.580745126+$ TSPOAP1-AS 1 * $(-1.587783887)+A C 005332.3 *(-0.324442618)$. After that, the respective patients were divided into high and low risk groups based on the median risk score of the training set in both the training and testing sets. Multivariate Cox analysis in the train set showed that age, tumor grade, and the risk score were independent risk factors associated with prognosis (Figure 4A). Multivariate Cox analysis in the test set also indicated that the risk score was an independent risk factor associated with prognosis (Figure 4B). The distributions of the risk score and survival status of training set and testing set are presented in Figure 5(training set: A, C, E and G; testing set: B, D, F and H). The Kaplan-Meier curve of OS and log-rank $t$ test was obtained in training set and testing set, patient's survival probabilities in the two datasets were statistically differed $(P<0.001$ in training set and $P=0.008$ in testing set) (Figure $5 E, F)$. The ROC predicting 5 -year survival probabilities in training set and testing set were presented in Figure $5 \mathrm{G}$ and Figure $5 \mathrm{H}$. The AUC for 1-year, 3-year and 5-year OS in training set was $0.861,0.829$ and 0.885 and in testing set was $0.708,0.785$ and 0.818 . Therefore, we constructed a prognostic risk nomogram based on the risk score (Figure 6).

\section{The clinicopathological factors, clusters and immune-scores associated with risk scores in PDAC}

Based on the median score of 11 prognostic m6A-regulated IncRNAs, 177 PDAC patients were divided into low-risk and high-risk groups. We analyzed the clinicopathological variables, cluster analysis, gene mutations and immune scores in the low-risk and high-risk groups in both the training and testing sets. The visualized clinical relevant heatmap based on 11-m6A regulated IncRNAs between low-risk and highrisk group is shown in Figure 10. With a difference in distribution between the low - and high-risk groups were the immune scores $(P<0.05)$ and cluster analysis $(P<0.001)$ (Figure $10 \mathrm{~A})$, which were higher in the low-risk group, and cluster analysis, which showed a denser distribution of Cluster 2 in the high-risk group. Patients with a low immune score, have an elevated risk and it follows that the immune score is favorable for prognosis. In terms of the distribution of clinicopathological characteristics and risk scores, patients with T3-T4 stage had an elevated risk compared with T1-T2 ( $P=0.016)$ (Figure 10B). PD-L1 expression was upregulated in the high-risk compared with the low-risk group $(P=0.037)$.

We parallel the Kaplan-Meier curve of OS among different age, gender, clinicopathological grade, TNM stage. As shown in Figure11, the OS curves showed distribution discrepancy in terms of all clinical variables and demographic characteristics except M1 and stage III-IV variables. Patients in the low-risk group of these variables all had a superior overall survival than those in the high-risk group (Figure 11A-F) $(P<0.01)$.

\section{Association of m6A-related IncRNAs with immunocytes}


We performed correlation analysis between the risk score and the 22 immune cells infiltrated in the TME, deleting samples with $\mathrm{P}<0.05$ for immune cells in TME. A total of 8 immune cells associated with the risk score were obtained, which were B cells naive, Plasma cells, CD8 T cells, T cells CD4 memory resting, Monocytes, Macrophages M0, Macrophages M1, and Dendritic cells activated. Positively correlated with the risk score were Dendritic cells activated, Macrophages M0, Macrophages M1, CD4 T cells (Figure 12AD). However, B cells naive, plasma cells, CD8 T cells, Monocytes were negatively correlated with the risk score (Figure 12E-H).

\section{Discussion}

Pancreatic ductal adenocarcinoma remains one of the most fatal malignancies, and the morbidity and mortality rates are increasing worldwide. Advances in surgical technology have provided opportunities for patients with localized resectable PDAC, while patients with metastatic urgently need more effective comprehensive therapy ${ }^{[26]}$. More distinctive, PDAC has a highly desmoplastic and immunosuppressive TME ${ }^{[27]}$. With the development of high-throughput sequencing, breakthroughs in the treatment of PDAC have been pursued at the molecular and cellular level, such as to explore novel molecular therapeutic targets ${ }^{[15]}$. Studies have found that the role of aberrant IncRNAs in the carcinogenicity of PDAC cannot

be ignored ${ }^{[28,29]}$. M6A modification is the most common epigenetic methylation modification of IncRNAs ${ }^{[30]}$. Previous studies have shown that m6A methylation modified IncRNAs can significantly affect the function of targets through RNA-protein interactions in a variety of tumor genes ${ }^{[31,32]}$. However, the function and mechanism of m6A-regulated IncRNAs in PDAC remain to be explored.

In this study, by performing gene differentially expression analysis in PDAC tumor tissues and normal tissues and co-expression analysis between 23 m6A RNA methylation regulators and IncRNAs by combined TCGA and GTEx databases, we identified 28 m6Aregulated IncRNAs correlated with prognosis. Based on this 28 m6A regulated IncRNAs, PDAC samples were categorized into Cluster 1 and Cluster 2 by consensus clustering analysis. In Cluster 1, the KM curves showed superior overall survival than Cluster 2 . However, in Cluster 2, CD276 expression was higher than in cluster 1. After finally screening 11 optimal prognosic correlated m6A regulated IncRNAs by LASSO regression analysis of 28 m6A-regulated IncRNAs, PDAC patients were divided into high-risk and low-risk groups according to the risk scores. Univariate and multivariate cox regression analysis of risk score, TNM stage, pathological grade, age, gender indicated that only risk scores were independent risk factors associated with prognosis. A prognostic risk nomogram was constructed based on the risk scores of $11 \mathrm{~m} 6 \mathrm{~A}$-regulated IncRNAs, and the AUC curve indicated the preferable accuracy and usefulness. Yuan and Hu constructed m6A-modified IncRNAs prognostic signature associated with overall survival, respectively ${ }^{[14,15]}$. Yuan's study enrolled only five m6A-modified IncRNAs, and Hu proposed a prognostic pathway based on four m6A-related IncRNAs. In our study, 11 m6A regulated IncRNAs that were the most relevant with prognosis were included according to LASSO regression analysis, based on which the prognostic model was constructed, which can reduce bias and exhibit the strongest correlation with prognosis. 
Immunotherapy has triggered a shift in treatment patterns in many solid tumors ${ }^{[33]}$. Due to the low mutational burden of PDAC tumor genes as well as the dense, inaccessible TME characteristic for fibrotic, hypoxic, and immunosuppressive properties renders it notoriously resistant to immunotherapy ${ }^{[34]}$. AntiPD-1 and anti-PD-L1 therapy has shown promising results in many solid tumors, but its efficacy in PDAC is limited ${ }^{[35,36]}$. A clinical trial study showing a 9.1 months median progression free survival (mPFS) and 15 months median OS in metastatic PDAC patients receiving concurrent anti-PD-1 therapy with gemcitabine / nab paclitaxel chemotherapy, which are the relatively desirable achieved by current anti-PD1 therapies in PDAC [37]. In the present study, although patients in the high-risk group had a higher level of PD-L1 gene expression than those in the low-risk group ( $\mathrm{P}=0.037), \mathrm{PD}-\mathrm{L} 1$ expression in PDAC tumor tissues and normal tissues did not differ. Therefore, it is urgent to exploit new immune checkpoints for PDAC to develop sensitive ICls or combination chemotherapy to achieve satisfactory treatment efficacy. The outcome that CD47, CD276 and ID01 genes were upregulated in PDAC tumor tissues compared with paracancerous tissues and had distribution differences $(P<0.05)$. Akane ${ }^{[38]}$ concluded that colorectal cancer (CRC) patients with upregulated CD47 expression have a poor prognosis and that antibodies against the CD47 immune checkpoint are effective for CRC. B7 homologous protein 3 (B7-H3, also known as CD276), a newly identified immunoregulatory protein member of the B7 family, which is also a novel target for tumor immunotherapy. CD276 is overexpressed in tumor tissues while limited expression is observed in normal tissues, and in TME plays an integral role ${ }^{[39]}$. Studies have confirmed CD276was upregulated in breast cancer, non-small cell lung and ovarian cancer tumor tissues ${ }^{[39]}$. Indoleamine 2,3 dioxygenase 1 (ID0-1), encoded by the ID01 gene on human chromosome 8p12, is the rate limiting enzyme in the conversion of tryptophan to kynurenine. Its expression is low in normal tissues and upregulated in a variety of tumors ${ }^{[40]}$. Overexpressed IDO1 is under interferon regulation inhibits apoptosis and promotes dormancy of tumor repopulating cells (TRCs) ${ }^{[41-43]}$. In our study, CD47, CD276 and IDO-1 were upregulated in PDAC tumor tissues compared with normal pancreatic tissues. It is therefore reasonable to suggest that the CD47, CD276 and ID01 genes serve as potential immune checkpoints in PDAC. Targeting them with immune checkpoint inhibitors or combined chemoradiotherapy might bring a new dawn to PDAC therapy.

PDAC samples were classified into cluster 1 and cluster 2 according to m6A regulated IncRNAs, for which GSEA analysis showed that the tumor-related signaling pathway was cell cycle in Cluster 2, which indicated a higher risk of tumor progression in Cluster 2. Meanwhile, Cluster 2 had a worse prognosis compared to Cluster 1 . This suggests a potential correlation between m6A regulated IncRNAs and tumor progression.

Based on 11 prognosis correlated m6A regulated IncRNAs, PDAC patients were divided into high-risk and low-risk groups, and patients in the high-risk group had a significantly worse long-term overall survival than those in the low-risk group. PD-L1 was significantly upregulated in the high-risk group. However, it was not possible to distinguish PDAC tumor tissues from normal tissues on PD-L1 expression. Therefore, surgery, chemoradiotherapy combined with potential ICls should be sought to synergistically against PDAC with a promising outcome to achieve. 


\section{Conclusion}

We exhaustively evaluated the m6A-regulated IncRNAs in relation to the PDAC tumor microenvironment, specific genetic mutations, differences in immune checkpoint expression, and prognosis. Therefore, highrisk PDAC patients can be distinguished, and furthermore, potential novel immune checkpoints may guide clinical drug development and immunotherapy.

\section{Materials And Methods}

\section{Data source}

We download the RNA transcription profiles and clinical information of PDAC patients from The Cancer Genome Atlas (TCGA) of UCSC Xena (https://xena.ucsc.edu/). Because the sample of paracancerous tissues in TCGA database was limited, the Genotype-Tissue Expression project (GTEx) database as a supplement to TCGA PDAC database. We downloaded the 167 gene profiles of PDAC normal samples from website https://xena.ucsc.edu/, whose gene expression was corrected and stored in FPKM form, which is consistent with the gene expression profile form in TCGA and processed in expression form. $\mathrm{R}$ package "sva" 1 was used to remove batch effects between TCGA and GTEx. The "sva"1 package supports the use of the "sva" function for the estimation of proxy variables and the "Combat" and "fsva" functions for the adjustment of batch and latent variables from known batch effects and prediction problems ${ }^{[16]}$. The RNA matrix file was divided into mRNA file and IncRNA data according to human genome annotation data. The correlation between 23 m6A regulator genes and IncRNAs was determined by the Pearson correlation coefficient. Absolute correlation coefficient $>0.4$ and $P$ value $<0.05$ was considered as $\mathrm{m} 6 \mathrm{~A}$ related IncRNAs.

\section{Construction of m6A methylation co-expression network with IncRNAs and consensus clustering analysis}

According to previous studies, $23 \mathrm{m6A}$ genes were extracted from TCGA, including expression data of "writers" (METTL3, METTL14, METTL16, WTAP, VIRMA, ZC3H13, RBM15, RBM15B), "readers" (YTHDC1, YTHDC2, YTHDF1, YTHDF2, YTHDF3, HNRNPC, FMR1, LRPPRC, HNRNPA2B1, IGFBP1, IGFBP2, IGFBP3, RBMX) and "erasers" (ALKBH5 and FTO) ${ }^{[17,18]}$. We then conducted co-expression analysis with R software. The "correlation coefficient $=$ " 0.4 " and "pvalueFilter $=0.001$ " were used as the parameters to select $m 6$ A regulated IncRNAs. The co-expression network was constructed by using "igraph" ${ }^{2} \mathrm{R}$ package. Through merged PDAC clinical information and m6A IncRNA expression data and performed univariate Cox regression analysis, the 28 m6A-regulated IncRNAs was determined, HR $>1$ indicates a risk factor and $\mathrm{HR}<1$ indicates benefit. The expression difference of m6A-regulated IncRNAs between tumor cohort and controlled cohort was revealed with boxplot and heatmap. The m6A-regulated IncRNA expression data was downloaded from TCGA. We used the "ConsensusClusterPlus" 3 package and clustering algorithm to classify PDAC patients into two clusters. The Kaplan-Meier analysis was performed to compare the overall survival (OS) of the two clustersby "survivalROC" 4 . 


\section{Construction of a prognostic risk model and evaluation the efficacy}

Least absolute shrinkage and selection operator (LASSO) regression analysis was selected to construct the prognosis risk signature of $\mathrm{m} 6 \mathrm{~A}$ regulated IncRNAs. Then we eliminate collinearity of the m6A regulated IncRNAs and avoid over-fitting of the constructed model. Subsequently, multivariate Cox regression analysis was utilized to construct $\mathrm{m} 6 \mathrm{~A}$ regulated IncRNAs prognosis signature. The risk score was calculated using the following formula: Riskscore $=\sum_{i=1}^{n} \boldsymbol{c o d} \boldsymbol{i} \boldsymbol{i} * \boldsymbol{x i}$

Where codfi is the coefficient and $\mathrm{Xi}$ is the transformed relative expression value of each selected IncRNA.Using this formula, each patient's risk score can be calculated, then the PDAC cohort was categorized into low-risk and high-risk groups based on median risk scores of training set. Through univariate and multivariate Cox regression analysis in training set, the clinical variables and $\mathrm{m} 6 \mathrm{~A}$ regulated IncRNAs associated with prognosis screened to construct the nomogram. The prognostic effect was assessed by area under the time-dependent ROC (t-ROC) analysis in the training, and testing set. The clinicopathological features were compared between low-risk and high-risk group and visualized in heatmap.

\section{Gene expression difference analysis and co-expression of PD-L1 and other ICs}

We select PD-L1 and several other potential immune checkpoint genes such as CD47, CD276 and ID01 [19-21] to visualize the distribution between normal and tumor, Cluster 1 and Cluster 2, low-risk and highrisk groups. The association between PD-L1 expression and m6A regulated IncRNAs was analyzed and visualized with R package "corrplot" 5 .

\section{Comparation of immune infiltration and Gene Set Enrichment Analysis (GSEA)}

Stromal, immune and estimate scores were calculated by using the ESTIMATE algorithm and the "estimate" ${ }^{6} \mathrm{R}$ package ${ }^{[22]}$. The differences between Cluster 1 and Cluster 2 were analysed. CIBERSORT deconvolution algorithm was used to obtain the abundance of tumor infiltrating 22 immune cells ${ }^{[23]}$. We use 1,000 permutations algorithm to calculate the final scores, the distribution of the immune infiltration of 22 immune cell subtypes between Cluster 1 and Cluster 2 groups was compared. The Gene Set Enrichment Analyses (GSEA) 4.1.0 was utilized to elaborate downstream Kyoto Encyclopedia of Genes and Genomes (KEGG) pathways in Cluster 1 and Cluster 2 group based on m6A-regulated IncRNAs subtype data. The c2.cp.kegg.v7.4.symbols.gmt was selected as the reference gene set database. The criteria for meeting the conditions are as follows: $\mathrm{P}<0.05$ and FDR<0.1. ${ }^{1}$ https://bioconductor.org/packages/release/bioc/html/sva.html ${ }^{2}$ https://cran.rproject.org/package=igraphdata

${ }^{3}$ https://bioconductor.org/packages/ConsensusClusterPlus/ ${ }^{4}$ https://cran.rproject.org/web/packages/survivalROC ${ }^{5}$ https://cran.rproject.org/package=corrplot ${ }^{6}$ https://bioinformatics. mdanderson.org/estimate/rpackage.html 


\section{Statistical analysis}

All statistical analysis is performed on R (version 4.0.4) software (https://www.r-project.org/) and multivariate Cox regression analysis was performed to determine the m6A-related IncRNAs associated with survival. The Kaplan-Meier curves were implemented to compare the different OS between the highrisk and low-risk groups according to distinct clinicopathological features. The Chi-square test or Fisher's exact test was used to analyse differences clinical information. The m6A IncRNA subtypes, clinicopathological features, risk scores, immune checkpoint expression, and immune infiltration were analyzed by a Pearson correlation test. A P value $<0.05$ was considered statistically significant. The flow chart can be seen in Figure 1.

\section{Abbreviations}

N6-methyladenosine: m6A, immune checkpoints : ICPs, pancreatic ductal adenocarcinoma : PDAC, Genotype-Tissue Expression project: GTE, The Cancer Genome Atlas: TCGA, least absolute shrinkage and selection operator : LASSO, Gene set enrichment analysis: GSEA, immune checkpoint blockade : ICB, tumor microenvironment :TME, Long non coding RNAs :IncRNAs, Cancer associated fibroblasts : CAFs, OS : overall survival, median progression free survival :mPFS, tumor repopulating cells :TRCs, timedependent ROC: t-ROC, Kyoto Encyclopedia of Genes and Genomes: KEGG, PD-1: programmed cell death protein 1, ;PD-L1: programmed cell death ligand 1

\section{Declaration Section}

\section{Acknowledgements}

We are grateful for the data from The Cancer Genome Atlas and Genotype-Tissue Expression project.

\section{Authors' contributions}

Wei Guo conceived, designed, and supervised the study. Guangzhen Qu performed data analysis and drafted the manuscript. Dong Wang and Weiyu Xu collected the data. All authors reviewed and approved the final manuscript.

\section{Funding}

None.

\section{Availability of data and materials}

The RNA sequencing profiles were downloaded from The Cancer Genome Atlas (TCGA) and GenotypeTissue Expression (GTEx) (“https://xenabrowser.net”)

\section{Ethics approval and consent to participate}


The study was approved by the Ethics Committee of Beijing Friendship Hospital of Capital Medical University.

\section{Competing interests}

The authors declare that they have no conflicts of interest.

\section{Consent for publication}

All authors have reviewed the manuscript and agree to publication.

\section{References}

1. Sung, H., et al., Global Cancer Statistics 2020: GLOBOCAN Estimates of Incidence and Mortality Worldwide for 36 Cancers in 185 Countries. CA Cancer J Clin, 2021. 71(3): p. 209-249.

2. Ferlay, J., C. Partensky, and F. Bray, More deaths from pancreatic cancer than breast cancer in the EU by 2017. Acta Oncol, 2016. 55(9-10): p. 1158-1160.

3. Siegel, R.L., K.D. Miller, and A. Jemal, Cancer statistics, 2020. CA Cancer J Clin, 2020. 70(1): p. 7-30.

4. Neuzillet, C., et al., State of the art and future directions of pancreatic ductal adenocarcinoma therapy. Pharmacol Ther, 2015. 155: p. 80-104.

5. Liang, C., et al., Complex roles of the stroma in the intrinsic resistance to gemcitabine in pancreatic cancer: where we are and where we are going. Exp Mol Med, 2017. 49(12): p. e406.

6. Delaunay, S. and M. Frye, RNA modifications regulating cell fate in cancer. Nat Cell Biol, 2019. 21(5): p. 552-559.

7. Qi, P., X.Y. Zhou, and X. Du, Circulating long non-coding RNAs in cancer: current status and future perspectives. Mol Cancer, 2016. 15(1): p. 39.

8. Wang, X., et al., Integrated TCGA analysis implicates IncRNA CTB-193M12.5 as a prognostic factor in lung adenocarcinoma. Cancer Cell Int, 2018. 18: p. 27.

9. Zheng, J., et al., Identification and validation of IncRNAs involved in m6A regulation for patients with ovarian cancer. Cancer Cell Int, 2021. 21(1): p. 363.

10. Hu, X., et al., IGF2BP2 regulates DANCR by serving as an N6-methyladenosine reader. Cell Death Differ, 2020. 27(6): p. 1782-1794.

11. Balachandran, V.P., G.L. Beatty, and S.K. Dougan, Broadening the Impact of Immunotherapy to Pancreatic Cancer: Challenges and Opportunities. Gastroenterology, 2019. 156(7): p. 2056-2072.

12. Sahai, E., et al., A framework for advancing our understanding of cancer-associated fibroblasts. Nat Rev Cancer, 2020. 20(3): p. 174-186.

13. Gotwals, P., et al., Prospects for combining targeted and conventional cancer therapy with immunotherapy. Nat Rev Cancer, 2017. 17(5): p. 286-301. 
14. Yuan, Q., et al., Development and validation of a novel N6-methyladenosine (m6A)-related multi- long non-coding RNA (IncRNA) prognostic signature in pancreatic adenocarcinoma. Bioengineered, 2021. 12(1): p. 2432-2448.

15. $\mathrm{Hu}, \mathrm{Y}$. and Y. Chen, N6-methylandenosine-related IncRNAs play an important role in the prognosis and immune microenvironment of pancreatic ductal adenocarcinoma. Sci Rep, 2021. 11(1): p. 17844.

16. Leek, J.T., et al., The sva package for removing batch effects and other unwanted variation in highthroughput experiments. Bioinformatics, 2012. 28(6): p. 882-3.

17. Tang, J., Q. Wan, and J. Lu, The prognostic values of m6A RNA methylation regulators in uveal melanoma. BMC Cancer, 2020. 20(1): p. 674.

18. Tian, S., et al., Regulation of Gene Expression Associated With the N6-Methyladenosine (m6A) Enzyme System and Its Significance in Cancer. Front Oncol, 2020. 10: p. 623634.

19. Papalampros, A., et al., Unique Spatial Immune Profiling in Pancreatic Ductal Adenocarcinoma with Enrichment of Exhausted and Senescent T Cells and Diffused CD47-SIRPa Expression. Cancers (Basel), 2020. 12(7).

20. Inamura, K., et al., Tumor B7-H3 (CD276) Expression and Survival in Pancreatic Cancer. J Clin Med, 2018. 7(7).

21. Blair, A.B., et al., IDO1 inhibition potentiates vaccine-induced immunity against pancreatic adenocarcinoma. J Clin Invest, 2019. 129(4): p. 1742-1755.22. Yoshihara, K., et al., Inferring tumour purity and stromal and immune cell admixture from expression data. Nat Commun, 2013. 4: p. 2612.

22. Yoshihara, K., et al., Inferring tumour purity and stromal and immune cell admixture from expression data. Nat Commun, 2013. 4: p. 2612.

23. Gentles, A.J., et al., The prognostic landscape of genes and infiltrating immune cells across human cancers. Nat Med, 2015. 21(8): p. 938-945.23. Gentles, A.J., et al., The prognostic landscape of genes and infiltrating immune cells across human cancers. Nat Med, 2015. 21(8): p. 938-945.

24. Hinshaw, D.C. and L.A. Shevde, The Tumor Microenvironment Innately Modulates Cancer Progression. Cancer Res, 2019. 79(18): p. 4557-4566.

25. Hui, L. and Y. Chen, Tumor microenvironment: Sanctuary of the devil. Cancer Lett, 2015. 368(1): p. 713.

26. Mizrahi, J.D., et al., Pancreatic cancer. Lancet, 2020. 395(10242): p. 2008-2020.

27. Koikawa, K., et al., Targeting Pin1 renders pancreatic cancer eradicable by synergizing with immunochemotherapy. Cell, 2021. 184(18): p. 4753-4771.e27.

28. Li, Z., et al., The long non-coding RNA HOTTIP promotes progression and gemcitabine resistance by regulating HOXA13 in pancreatic cancer. J Transl Med, 2015. 13: p. 84.

29. Hu, X., et al., IGF2BP2 regulates DANCR by serving as an N6-methyladenosine reader. Cell Death Differ, 2020. 27(6): p. 1782-1794.

30. Fu, Y., et al., Gene expression regulation mediated through reversible m $₫ A$ RNA methylation. Nat Rev Genet, 2014. 15(5): p. 293-306. 
31. Ferrè, F., A. Colantoni, and M. Helmer-Citterich, Revealing protein-IncRNA interaction. Brief Bioinform, 2016. 17(1): p. 106-16.

32. Tu, Z., et al., N6-Methylandenosine-Related IncRNAs Are Potential Biomarkers for Predicting the Overall Survival of Lower-Grade Glioma Patients. Front Cell Dev Biol, 2020. 8: p. 642.

33. Timmer, F.E.F., et al., Pancreatic Cancer and Immunotherapy: A Clinical Overview. Cancers (Basel), 2021. 13(16).

34. Huber, M., et al., The Immune Microenvironment in Pancreatic Cancer. Int J Mol Sci, 2020. 21(19).

35. Hodi, F.S., et al., Nivolumab plus ipilimumab or nivolumab alone versus ipilimumab alone in advanced melanoma (CheckMate 067): 4-year outcomes of a multicentre, randomised, phase 3 trial. Lancet Oncol, 2018. 19(11): p. 1480-1492.

36. Motzer, R.J., et al., Nivolumab versus Everolimus in Advanced Renal-Cell Carcinoma. N Engl J Med, 2015. 373(19): p. 1803-13.

37. Weiss, G.J., et al., Phase Ib/Il study of gemcitabine, nab-paclitaxel, and pembrolizumab in metastatic pancreatic adenocarcinoma. Invest New Drugs, 2018. 36(1): p. 96-102.

38. Sugimura-Nagata, A., et al., Expression and Prognostic Significance of CD47-SIRPA Macrophage Checkpoint Molecules in Colorectal Cancer. Int J Mol Sci, 2021. 22(5).

39. Zhou, W.T. and W.L. Jin, B7-H3/CD276: An Emerging Cancer Immunotherapy. Front Immunol, 2021. 12: p. 701006.

40. Liu, M., et al., Targeting the IDO1 pathway in cancer: from bench to bedside. J Hematol Oncol, 2018. 11(1): p. 100.

41. Liu, Y., et al., Blockade of IDO-kynurenine-AhR metabolic circuitry abrogates IFN-Y-induced immunologic dormancy of tumor-repopulating cells. Nat Commun, 2017. 8: p. 15207.

42. Liu, Y., et al., STAT3/p53 pathway activation disrupts IFN- $\beta$-induced dormancy in tumor-repopulating cells. J Clin Invest, 2018. 128(3): p. 1057-1073.

43. Liu, Y., et al., Tumor-Repopulating Cells Induce PD-1 Expression in CD8(+) T Cells by Transferring Kynurenine and AhR Activation. Cancer Cell, 2018. 33(3): p. 480-494.e7.

\section{Figures}




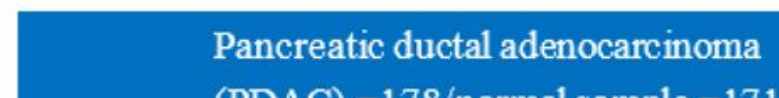

$(\mathrm{PDAC})=178 /$ normal sample $=171$

RNA transcriptome profiling data

(TCGA combined GTEx)

$23 \mathrm{~N}-6$ methyladenosine

methylation regulators

28 m6A-related IncRNAs

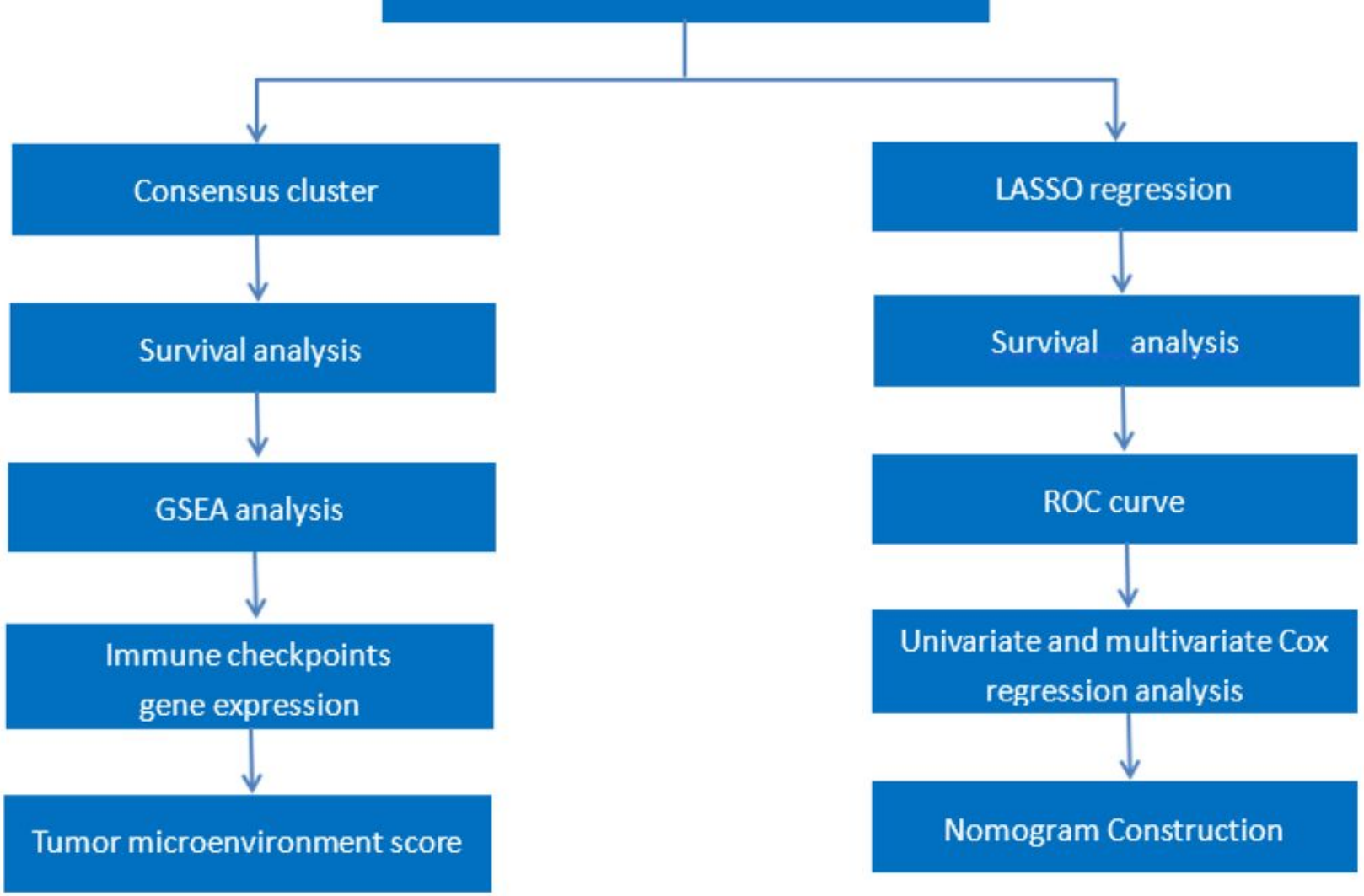

\section{Figure 1}

\section{Figure 1}

The flow chart of this study. 

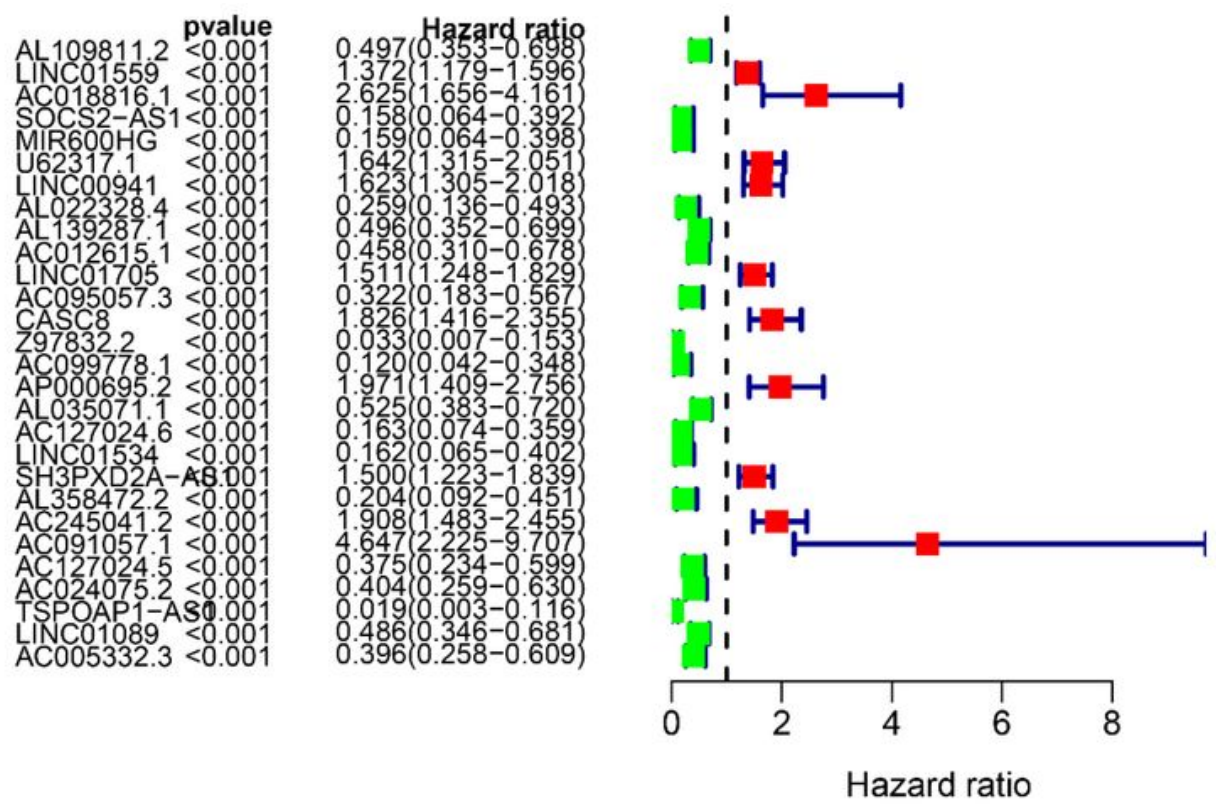

Figure 2A

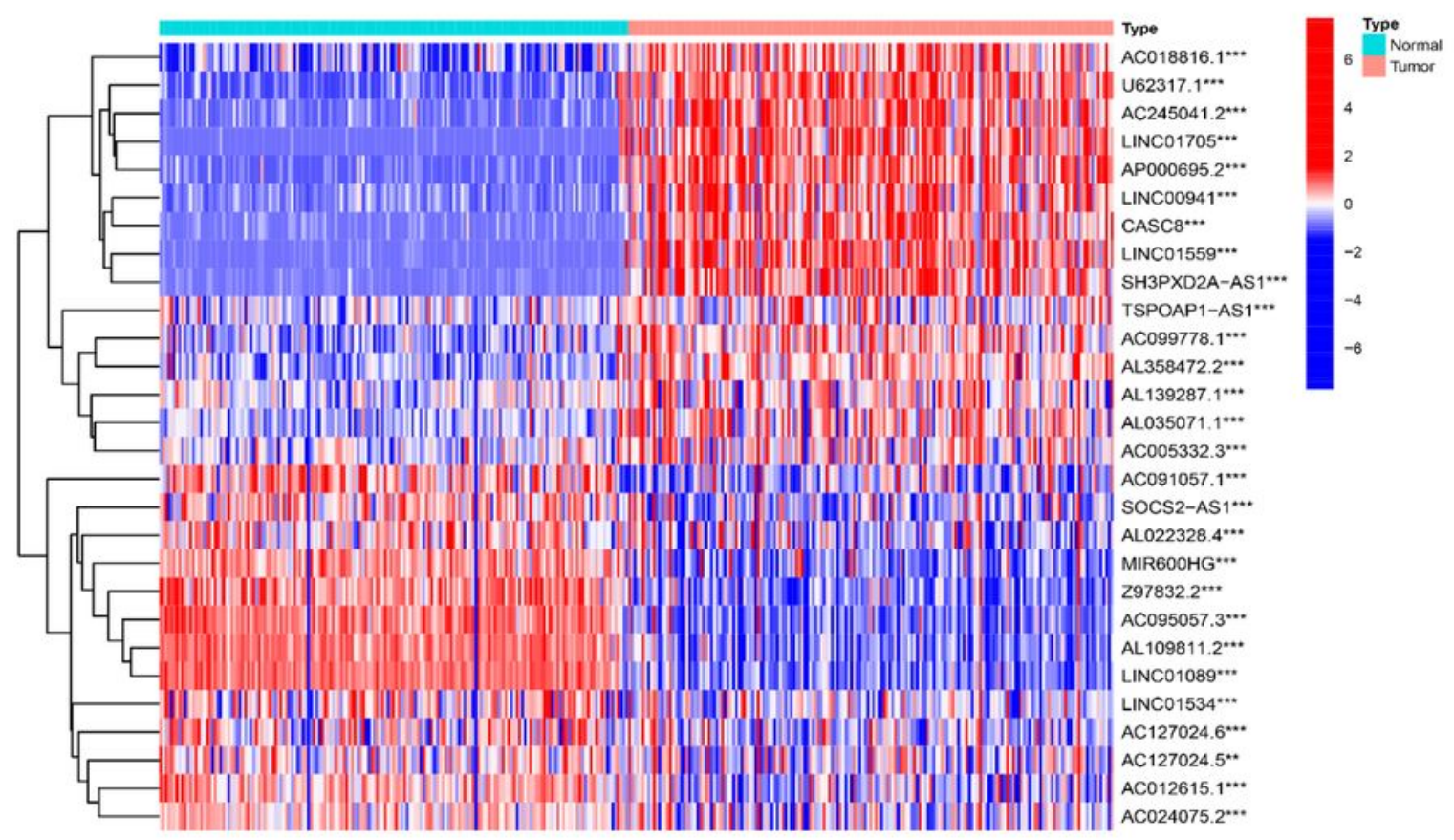

Figure 2B

Figure 2

M6A RNA methylation-related IncRNAs regulators in PDAC samples. (A) Univariate Cox regression was conducted to select the 28 m6A RNA methylation-related IncRNAs. (B) Heatmap presented the overall expression of 28 m6A-related IncRNAs in PDAC tissues and normal tissues from The Cancer Genome

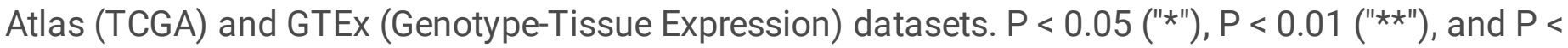
$0.001(" \star \star \star ")$. 


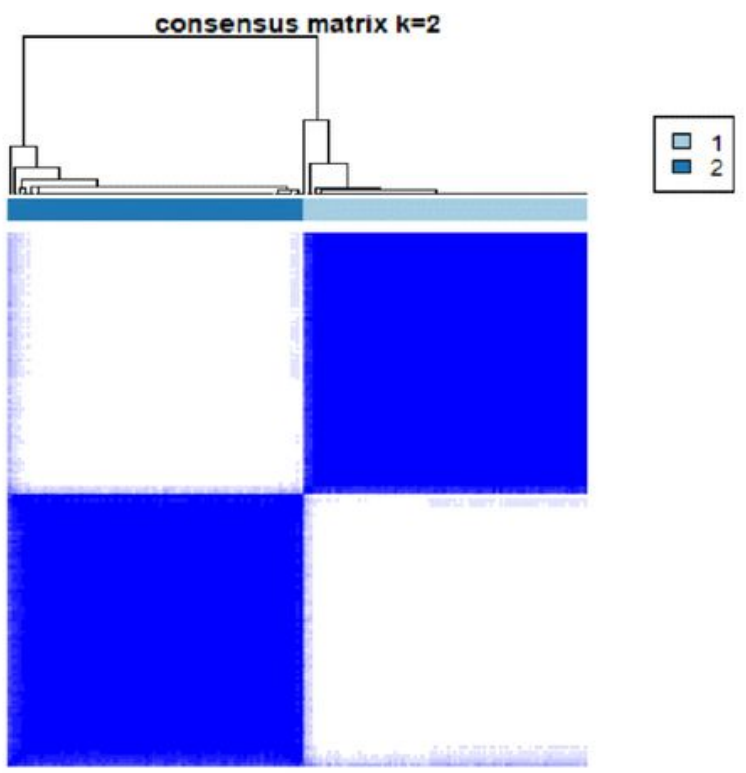

Figure 3A
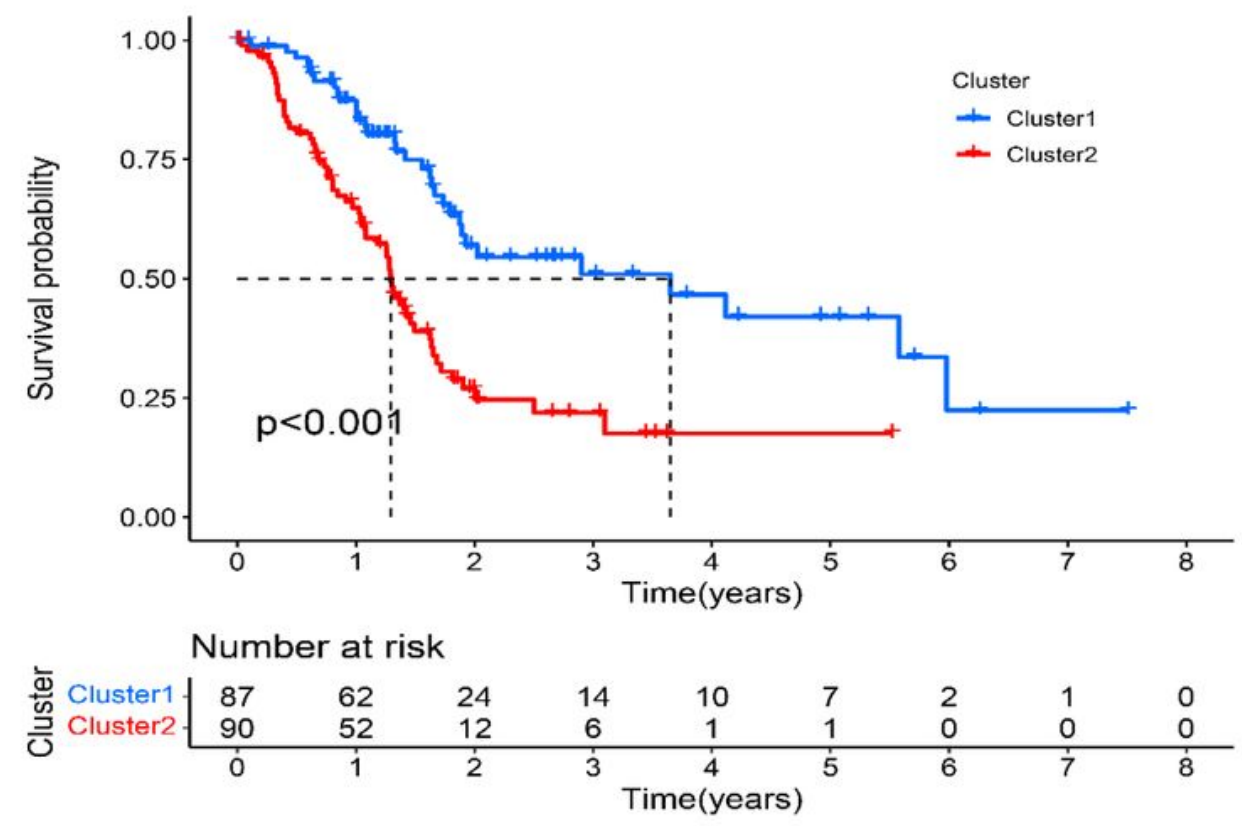

Figure 3B

\section{Figure 3}

Association between m6A-related IncRNAs and survival probability (A): Results of consensus clustering analysis at $k=2$ (B): Kaplan-Meier curve of overall survival probabilities in different clusters of PDAC samples 


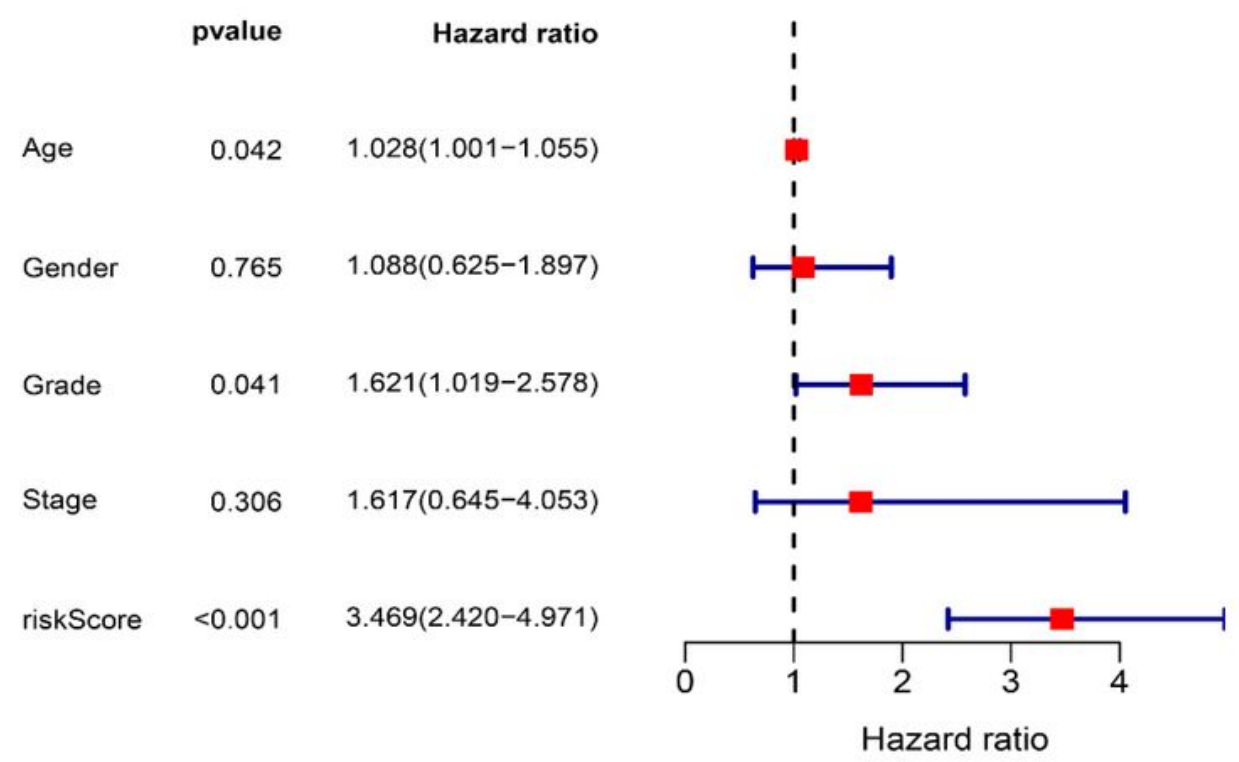

Figure 4A

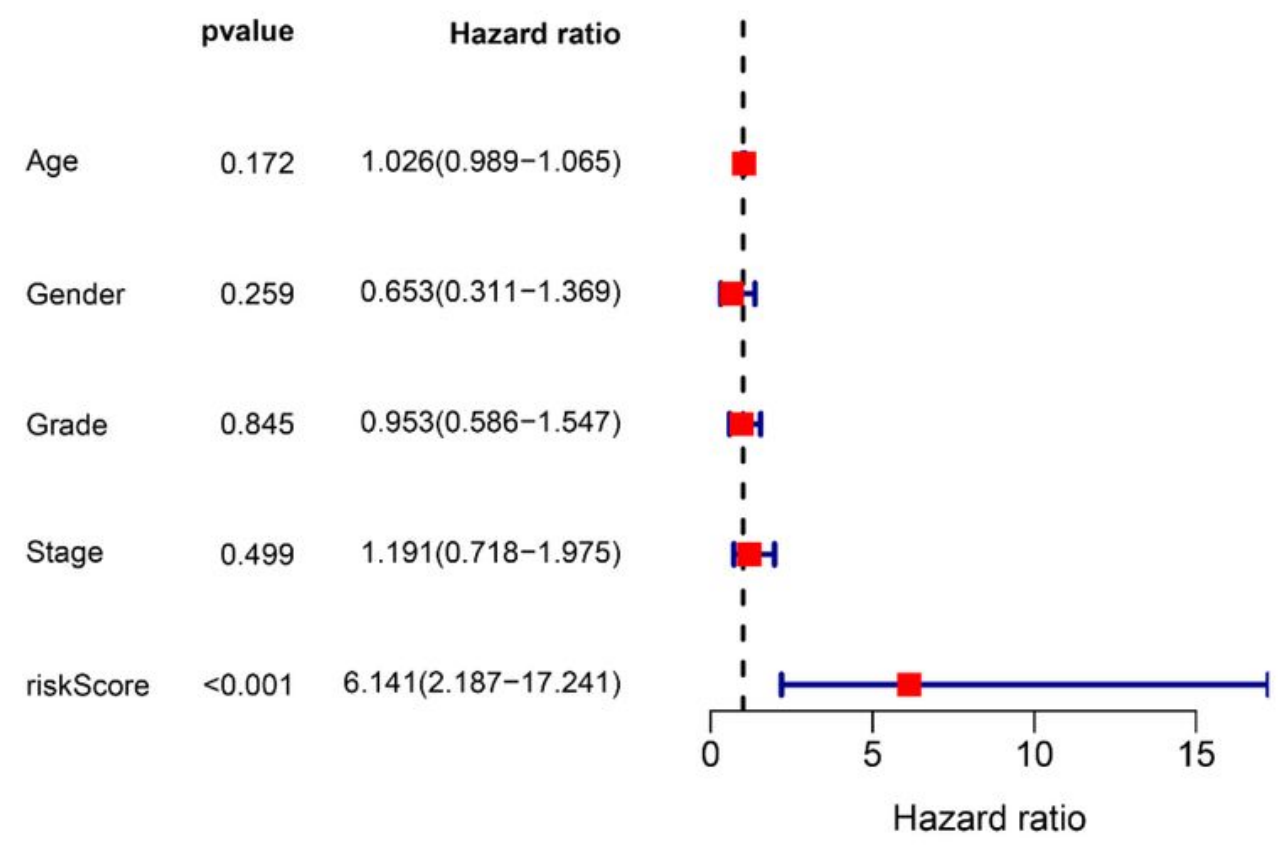

Figure 4B

\section{Figure 4}

Multivariate Cox regression analysis was performed to screen independent risk factors associated with prognosis. (A): Results of multivariate Cox regression analysis in the training set (B): Multivariate Cox regression analysis in the testing set 


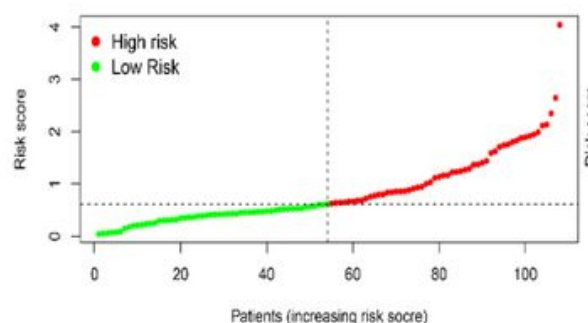

Figure 5A

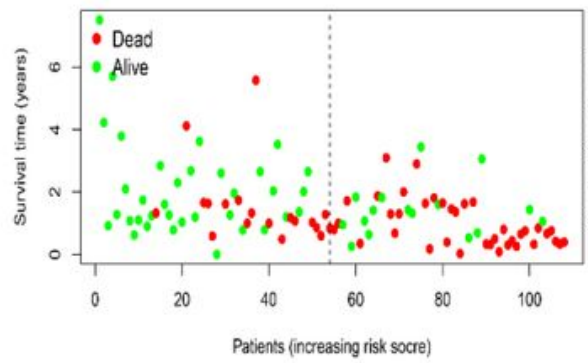

Figure 5C
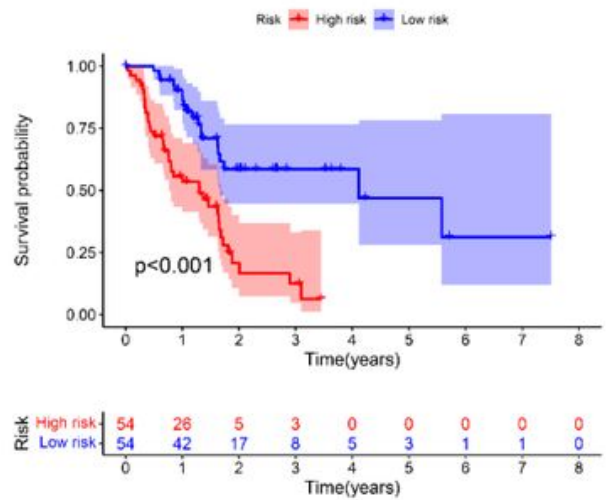

Figure 5E

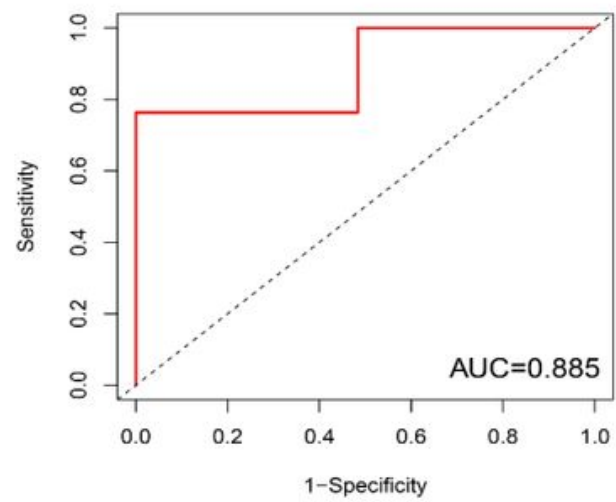

Figure 5G

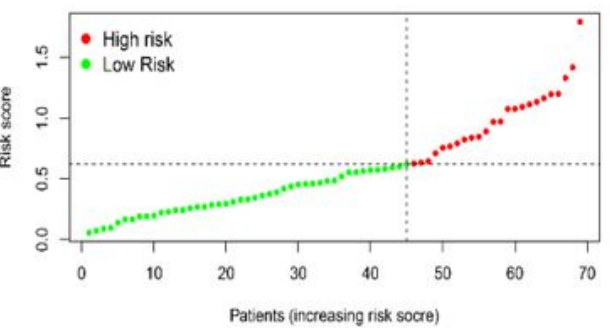

Figure 5B

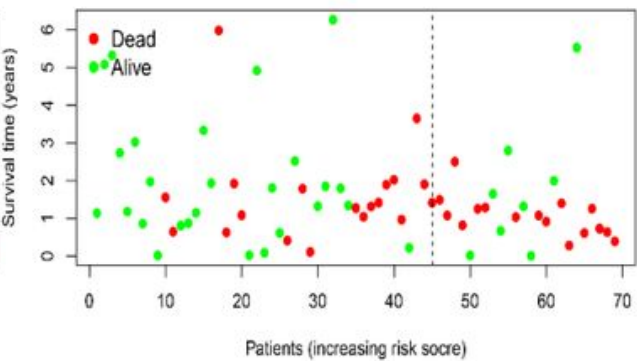

Figure 5D
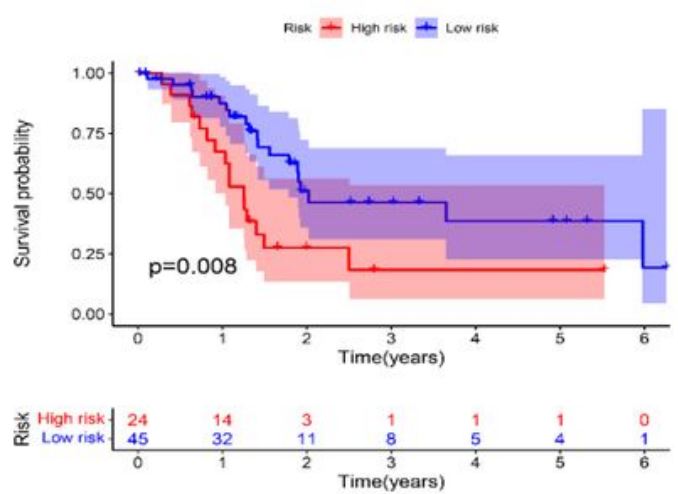

Figure 5F

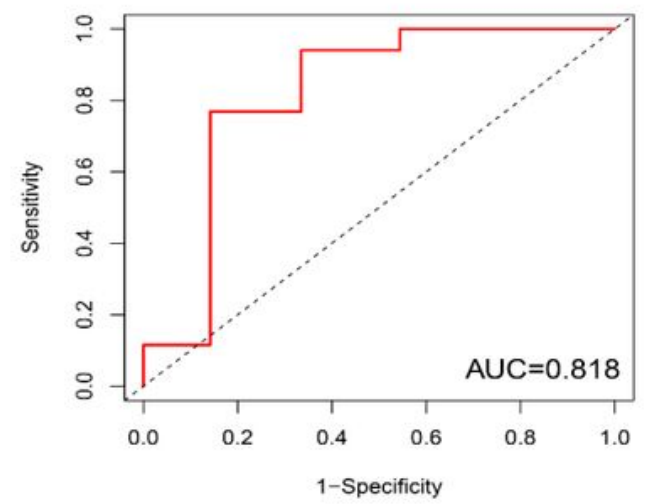

Figure 5H

\section{Figure 5}

Construction and validation of prognostic risk model of m6A-related IncRNAs. Heatmap of distribution of risk score based on eleven prognostic signatures in the train cohort ( $A, C)$ and test cohort (B, D). KaplanMeier curves of OS for patients with PDAC according to the risk score in the train cohort (E) and test cohort (F). ROC curves predicting the value for 5-years and area under curve (AUC) of the risk score in the train cohort (G) and test cohort $(H)$. 


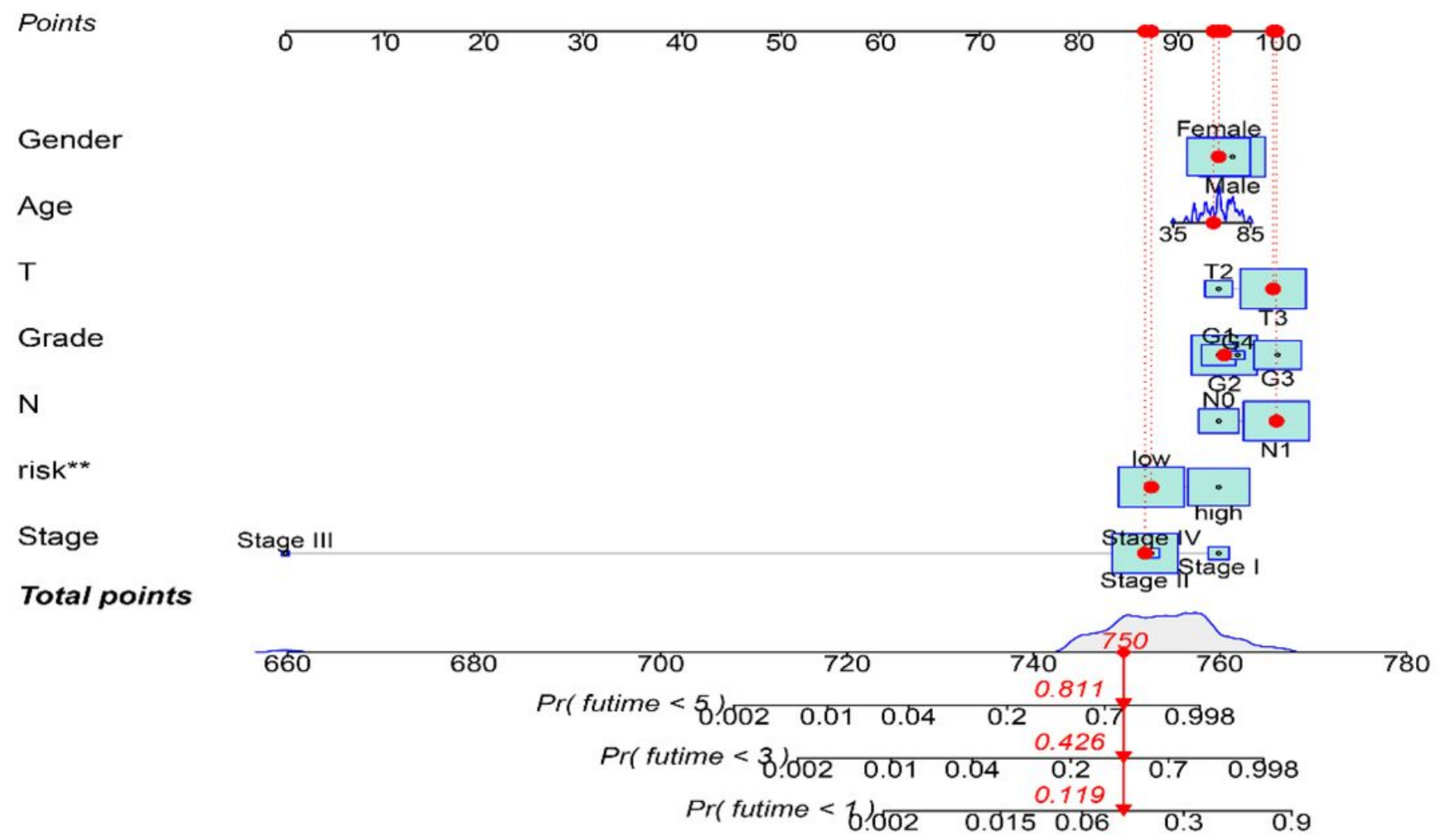

\section{Figure 6}

\section{Figure 6}

The risk prognosis nomogram constructed by the risk score to predict the $1-, 3$-, and 5-year overall survival (OS) probabilities of PDAC patients 

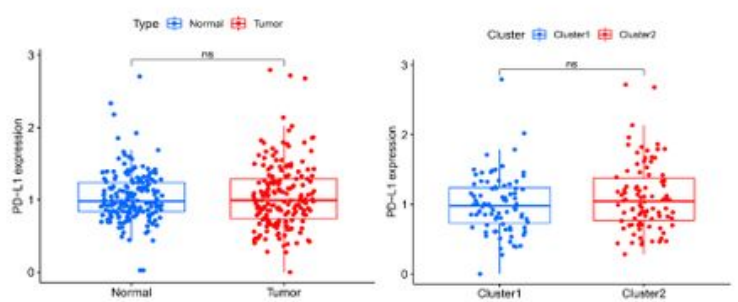

Figure 7A

Figure 7B
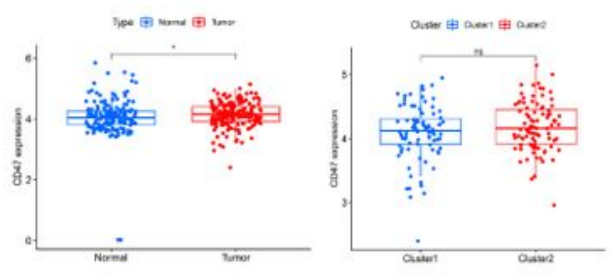

Figure 7C

Figure 7D
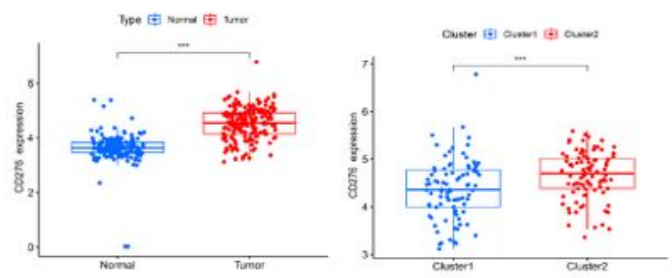

Figure 7E

Figure 7F
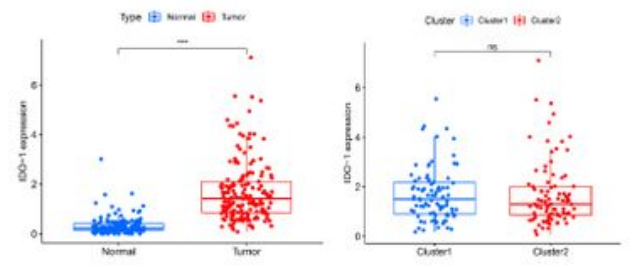

Figure 7G

Figure 7H

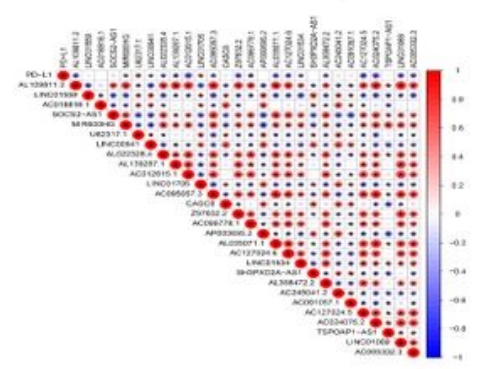

Figure 7I

\section{Figure 7}

Expression of PD-L1 and co-expression analysis and other potential immune checkpoint genes between PDAC tumor and normal tissues and between Cluster 1 and Cluster 2 (A-I): Expression contrasts between PDAC tumor and normal tissues and between Cluster 1 and Cluster 2 of PD-L1 (A, B), CD47 (C, D) , CD276 $(\mathrm{E}, \mathrm{F}), \mathrm{ID01}(\mathrm{G}, \mathrm{H})$ and co-expression analysis (I). 


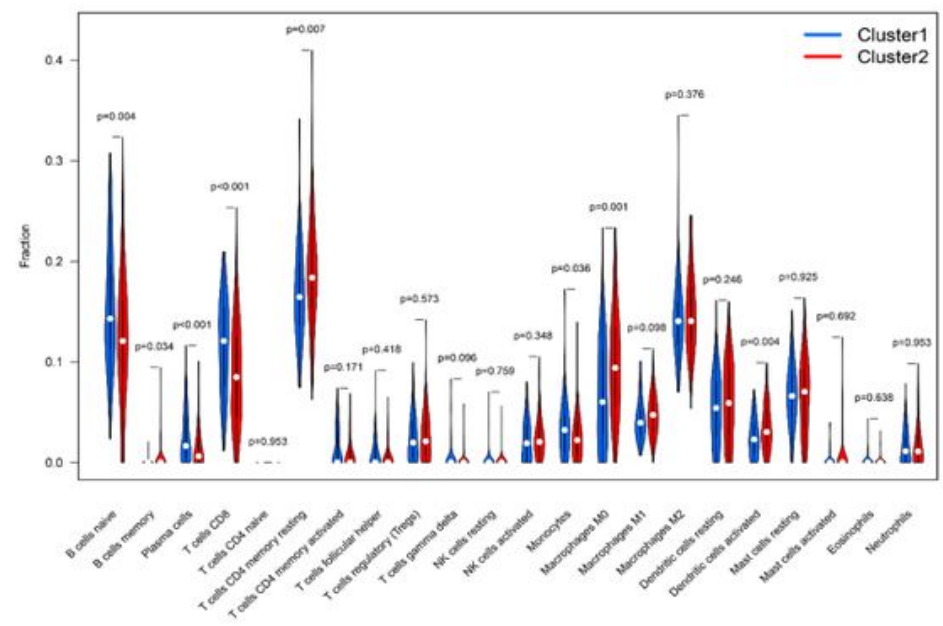

Figure 8A
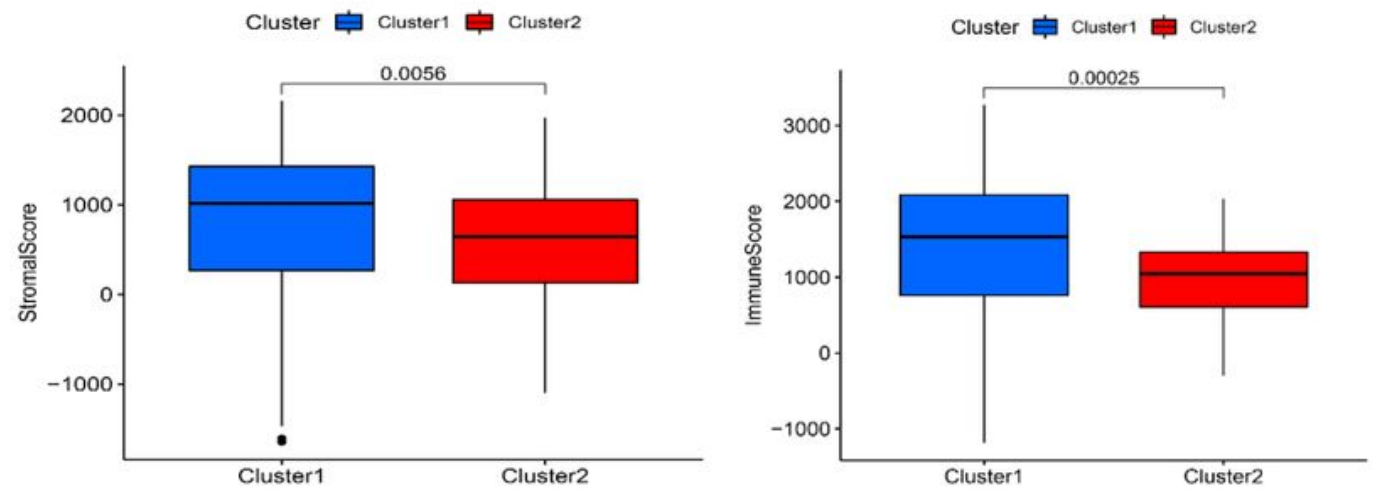

Figure 8B

Figure 8C

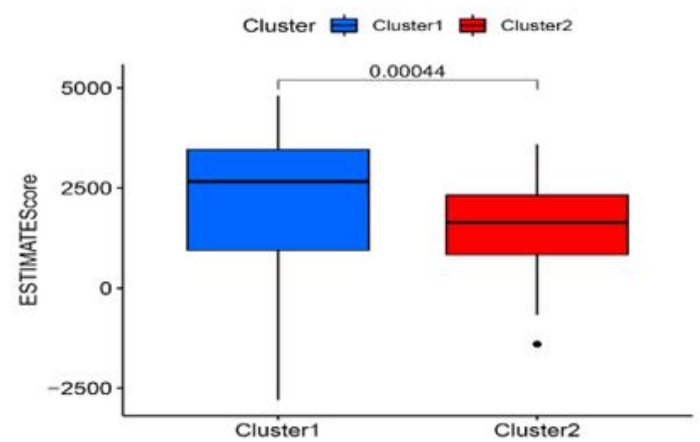

Figure 8D

\section{Figure 8}

Immune cell infiltration and Stroma scores, Immune score and ESTIMATE score in tumor microenvironment (A): The infiltration degree of 22 immune cells in the tumor microenvironment between different subgroups. (B): Stromal score, (C): Immune score and (D) ESTIMATE score in two clusters 
Enrichment plot: KEGG_CELL_CYCLE

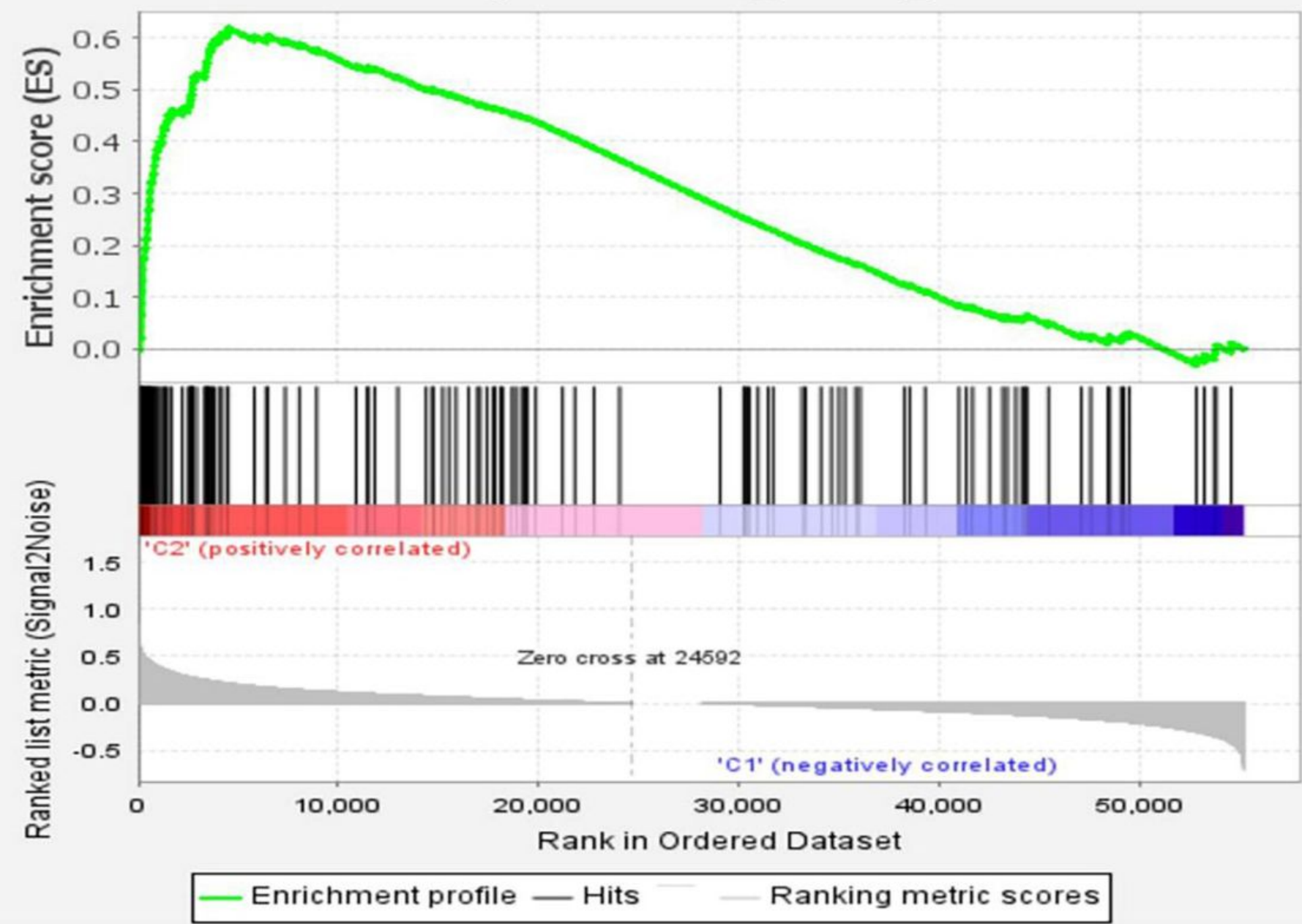

\section{Figure 9}

Figure 9

Gene set enrichment analysis (GSEA) and genes mutation analysis were performed to predict the potential functions and pathways between the two clusters. 


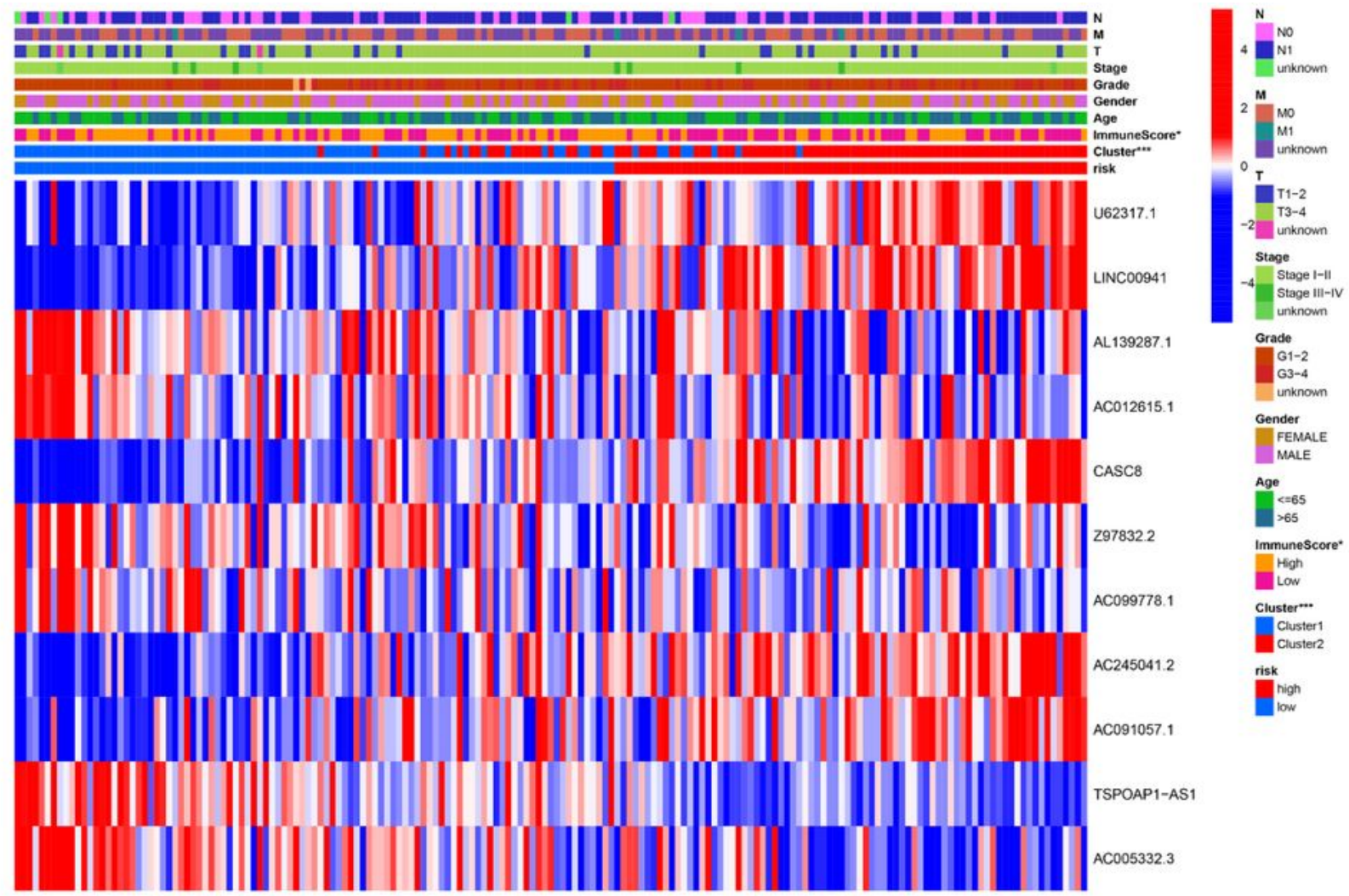

Figure 10A

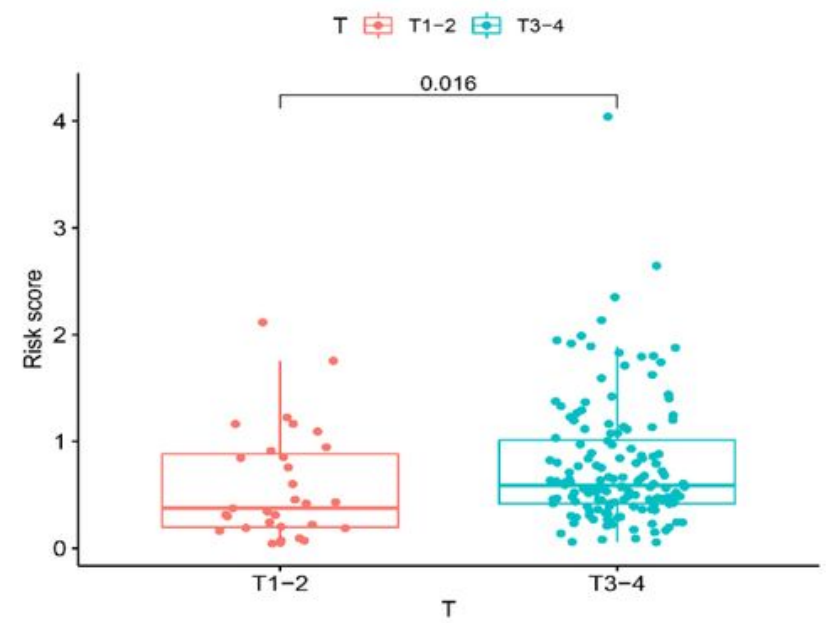

Figure 10B

\section{Figure 10}

Association between the m6A-related IncRNAs and clinicopathological and prognostic features of the PDAC patients A: The correlation of the two clusters with clinicopathologic features was visualized by

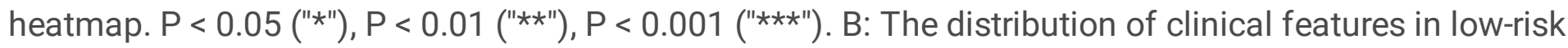

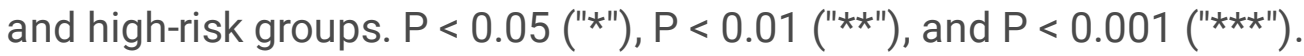


Patients with G1-2

Risk + high + low
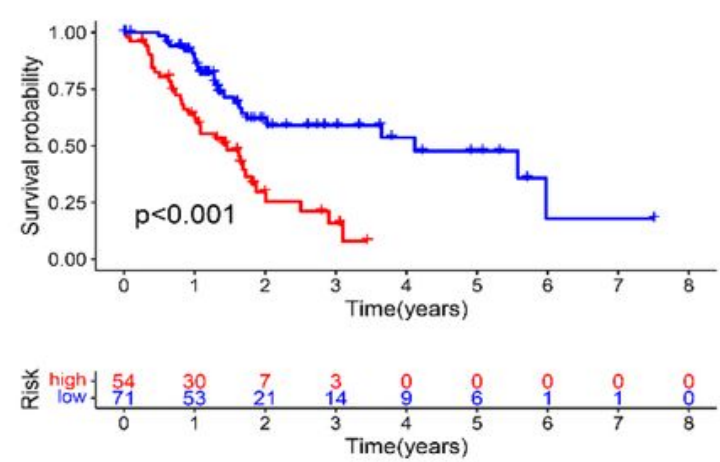

Figure 11A

Patients with Stage I-II
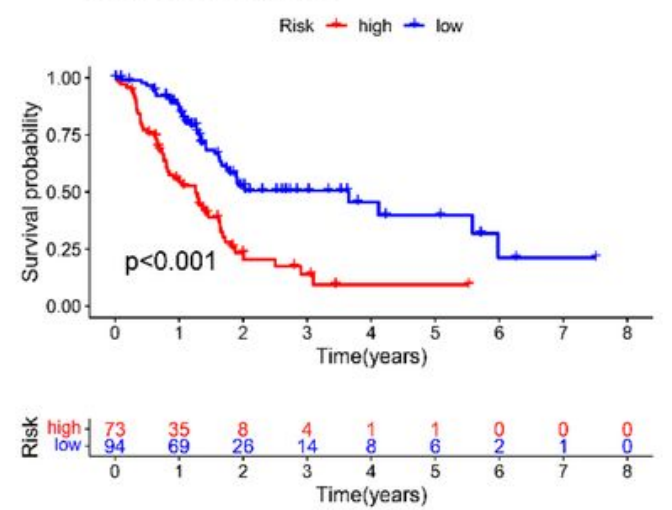

Figure $11 \mathrm{C}$

Patients with NO

Risk + high + low
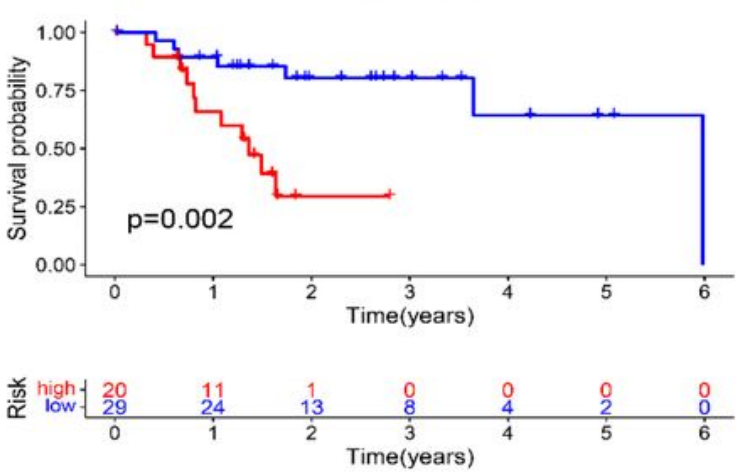

Figure 11E
Patients with G3-4

Risk + high + low
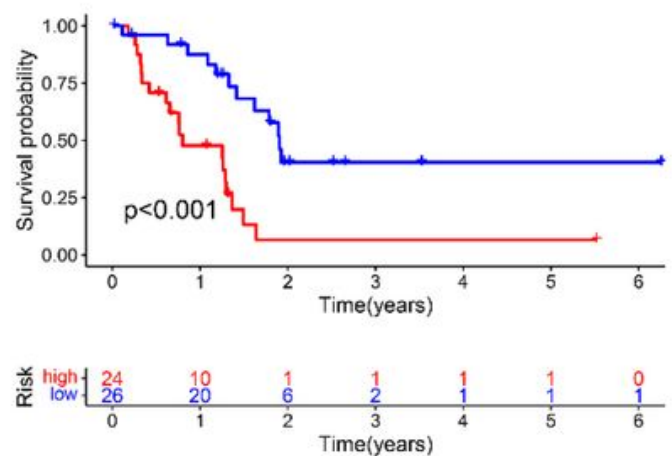

Figure 11B

Patients with T3-4

Risk + high + low
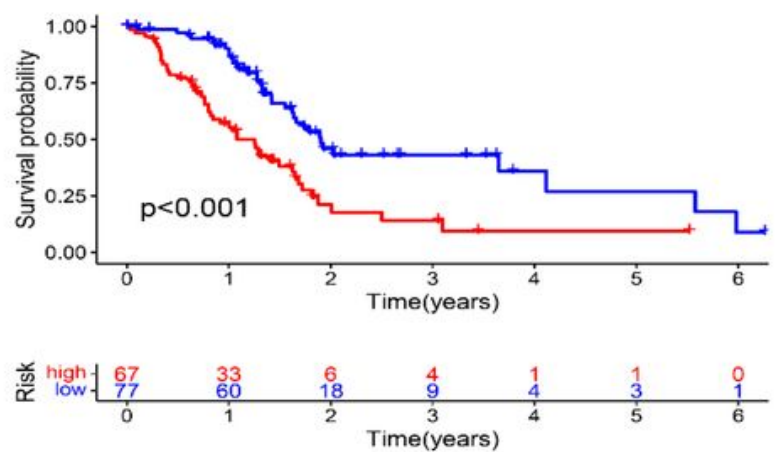

Figure 11D

Patients with MO

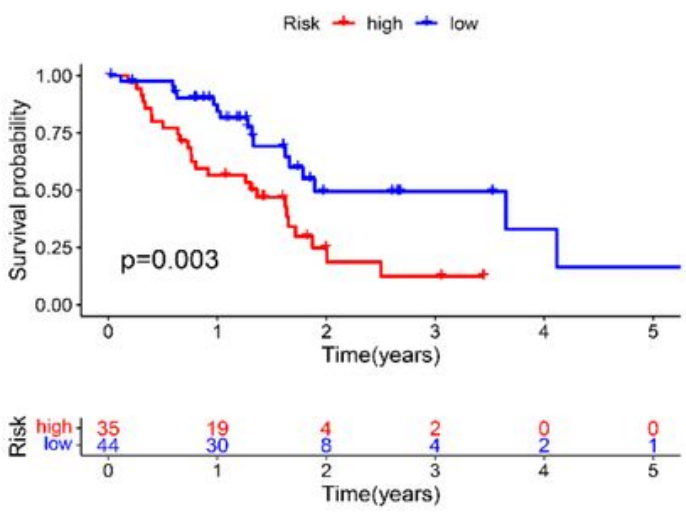

Figure 11F

\section{Figure 11}

The visualized Kaplan-Meier curves regarding the differences in overall survival between high-risk and low-risk groups in terms of pathological grade (A, B), tumor stage (C), T stage (D), N stage (E), and M stage $(\mathrm{F})$. 


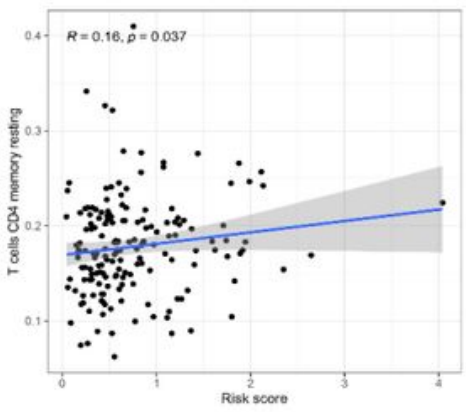

Figure 12A

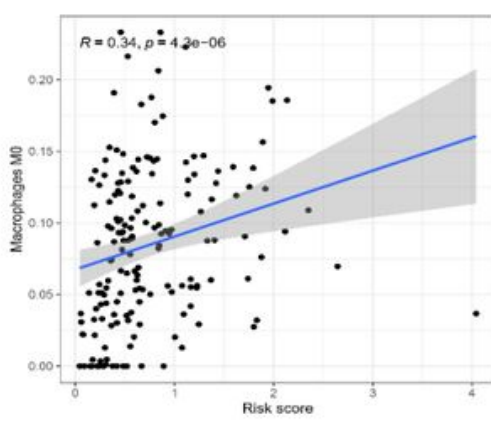

Figure 12C

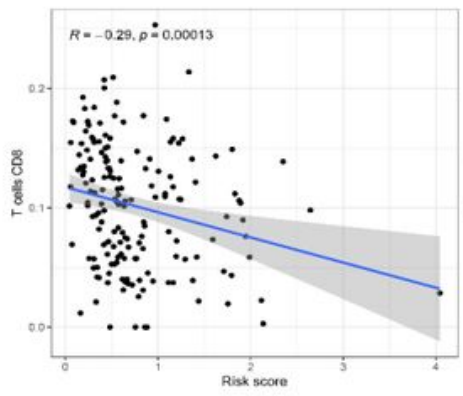

Figure 12E

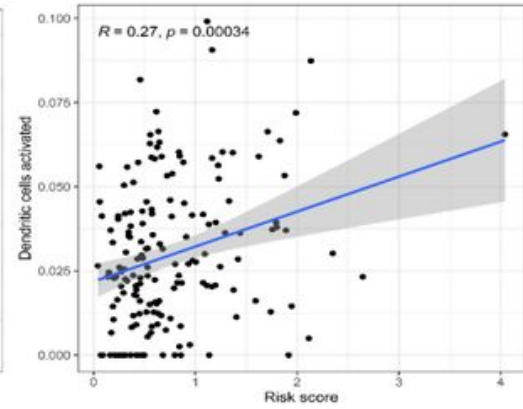

Figure 12B

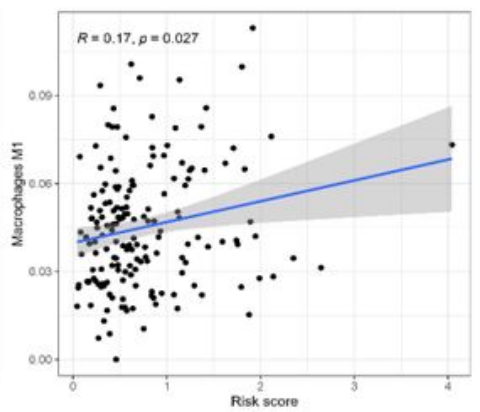

Figure 12D

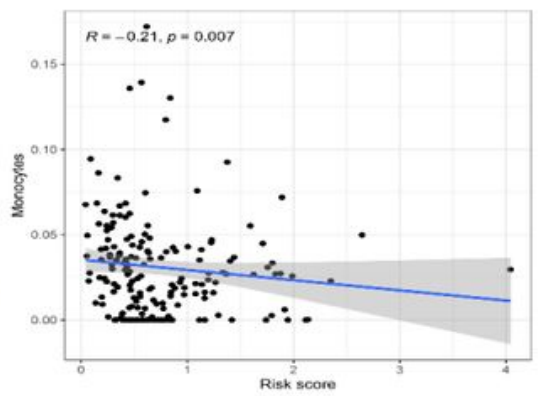

Figure 12F

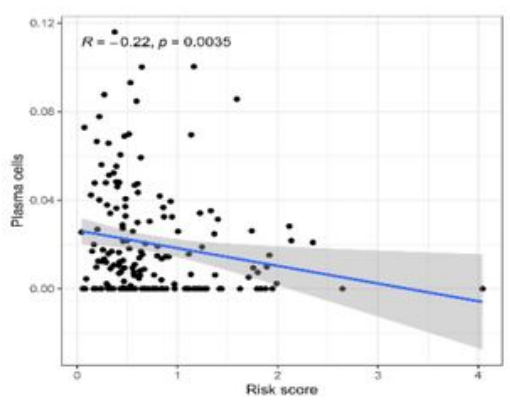

Figure 12G

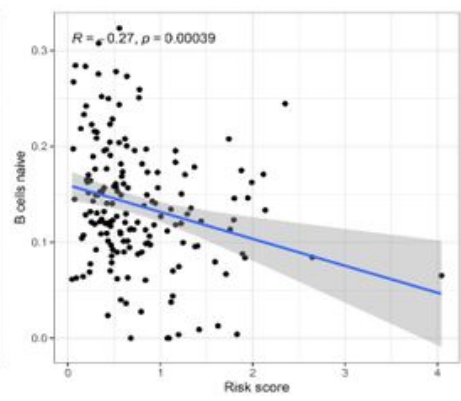

Figure 12H

\section{Figure 12}

Correlation between risk score and degree of infiltration for 8 immune cells. (A-H) T cell CD4 memory resting (A), Dendritic cells activated (B), Macrophage M0 (C), Macrophage M1 (D), T cell CD8 (E), Monocytes (F), Plasma cells (G), and B cells naïve $(H)$

\section{Supplementary Files}


This is a list of supplementary files associated with this preprint. Click to download.

- Supplements.docx 